\begin{tabular}{lcr}
\hline \multicolumn{3}{c}{ A N N A L ES } \\
UNIVERSITATIS M A R I A E C URIE-SKŁ ODOWS K A \\
LOL. XXXVI & SECTIOFF & $1-2018$ \\
\hline
\end{tabular}

\title{
SAFA ZOUAIDI
}

ORCID ID: https://orcid.org/0000-0003-1366-6808

Université Grenoble Alpes, Université de Gabès

\section{Associations sémantiques et syntaxiques : à l'exemple des combinaisons des verbes d'émotion dans les champs de la colère et de l'admiration en français et en arabe}

\begin{abstract}
Łączliwość składniowa i leksykalno-semantyczna czasowników odnoszących się do stanów emocjonalnych w polu semantycznym gniewu, złości i podziwu - na materiale języka francuskiego i arabskiego
\end{abstract}

Semantic and syntactic associations: On the example of the combinations of the verbs of emotion in the fields of anger and admiration in French and Arabic

\section{INTRODUCTION}

La question principale de notre étude concerne les correspondances entre le français et l'arabe dans l'expression de l'émotion ' : deux verbes d'émotion (V_émotion) (énerver et son correspondant [Caydaba $]^{2}$ et deux verbes de sentiment (admirer et son correspondant [?ą̧aba]). Les V_émotion sont difficiles à classer à cause de leur polysémie (frapper, éblouir) (Bouchard, 1995). Certains travaux, cherchant à circonscrire cette classe, pour le français, s'appuient sur des critères syntaxiques

1 Je remercie Mme Iva Novakova pour m'avoir appris que la comparaison entre les langues faisait apparaître des particularités linguistiques qui ne ressortiraient pas nécessairement d'une analyse unilingue et pour ses remarques pertinentes lors de l'élaboration de ce travail.

${ }^{2}$ Les lexèmes arabes seront présentés en trois lignes dans le but de les rendre plus commodes, surtout pour les locuteurs non natifs : une première ligne de translittération conformément à l'alphabet phonétique international (API) (Orig.translit.), puis une deuxième ligne de traduction en français du mot original en arabe en vue de gloser les associations verbales selon les règles du Leipzig Glossing (Trad.litt.). La troisième ligne concerne la traduction française des exemples arabes et permet de mettre en œuvre la comparaison (Trad.fr.). 
(Dubois et Dubois-Charlier, 1997; Harris, 1988; Ibrahim, 2001). D'autres partent, à l'inverse, d'ensembles sémantiquement similaires de verbes afin d'aborder leurs propriétés syntaxiques (Mathieu 2000; Ruwet, 1972, 1994). Nous remarquons, ici, l'absence d'analyses contrastives intégrant les deux niveaux d'analyse: sémantique et syntaxique.

Concernant l'arabe, les difficultés sont d'une autre nature. Les travaux dans ce domaine sont beaucoup moins nombreux (Arbaoui, 2010; El Kassas, 2007) et il n'existe pas, à notre connaissance, d'études sémantico-syntaxique des V_émotion $^{3}$. La notion même de collocation est traitée, jusque là, dans une perspective contrastive anglais-arabe sous un angle strictement lexicographique (Abu-Ssaydeh, 2007). Cela constitue, certes, une difficulté, mais justifie pleinement la recherche contrastive entreprise qui est, dans ce sens, novatrice. Notre objectif consiste à mieux appréhender les spécificités de la combinatoire des V_d'émotion à travers la structuration de ce champ sémantique dans les deux langues par rapport à ce que propose la littérature linguistique en syntaxe et en sémantique contrastive.

Après une présentation du cadre théorique, de l'approche contrastive et de la méthodologie, ainsi que des corpus (Emolex ${ }^{4}$ et Arabicorpus ${ }^{5}$ ), nous proposerons une analyse sémantico-syntaxique de ces associations et ce dans les deux langues comparées.

\section{CADRE THÉORIQUE, MÉTHODOLOGIE, CORPUS}

Notre travail s'appuie principalement sur la théorie du Lexical priming de Michael Hoey (2005). Cette théorie, qui appartient au contextualisme britannique, postule que les mots ont des «préférences» (admirer secrètement) ou des «aversions» (*énerver secrètement) envers d'autres mots ou position syntaxiques. Les associations sémantiques entre les mots sont amorcées par les locuteurs en fonction de degrés d'attirance entre eux et aussi du savoir linguistique des locuteurs. Notre

${ }^{3}$ Nous avons trouvé quelques remarques (Vernier, 1892), mais elles sont parcellaires et insuffisantes pour servir d'appui à notre étude.

${ }^{4}$ Le projet franco-allemand ANR/DFG Emolex (ANR-09-FASHS-017), sous la responsabilité d'I. Novakova (Université Stendhal, Grenoble Alpes) et de P. Blumenthal (Université de Cologne), a pour objectif l'étude du lexique des émotions dans cinq langues européennes dans ses aspects sémantique, syntaxique et discursif. Le corpus journalistique est composé de 120 millions mots issus des journaux Le Monde, Le Figaro, Libération et Ouest-France parus en 2007 et 2008. Http:// emolex.u-grenoble3.fr/emoBase/ (23.02.2018).

${ }^{5}$ Le corpus journalistique est un corpus en ligne avec des fonctionnalités en anglais : http:// arabicorpus.byu.edu/ (20.02.2018). Il est constitué de 130 millions de mots. Il s'agit des journaux Al-Masri Al-yawm 2010, Ahram (1999), Churuq, Al-ghad01, Alghad02, Hayat (1997), Hayat (1996), Tajdid (2002), Watan (2002), Thawra. 
étude se base également sur l'analyse de la combinatoire syntaxique et lexicale (Novakova et Tutin, 2009). L'association lexicale sera utilisée au sens de Hausmann (1989), c'est-à-dire comme étant une collocation, une combinaison phraséologique binaire composée d'une base (V_pivot) et d'un collocatif (par exemple: énerver prodigieusement, admirer secrètement).

Pour y parvenir, notre recherche s'appuie, d'un point de vue méthodologique, sur une démarche quantitative et qualitative et privilégie la démarche contrastive (Creissels, 1995). Il s'agit, ainsi, d'un travail contrastif fondé sur corpus (Sinclair, 1991) dans lequel les données sont recueillies grâce à des indices statistiques 6 . Notre point de départ est de considérer que les accompagnateurs, statistiquement spécifiques de ces verbes renseignent sur différentes dimensions sémantiques comme l'intensité, l'aspect.

Pour ce faire, nous nous appuyons sur des textes journalistiques pour le français qui sont issus du corpus (Emolex l'interface EmoConc). Pour l'arabe, nous avons utilisé l'Arabicorpus, équilibré en nombre de mots et en types de textes. Le tableau suivant répertorie l'ensemble des verbes étudiés et indique le nombre total d'associations pivot-collocatif pour chaque champ:

Tableau 1. Nombre d'association V_pivot-collocatif en français et en arabe

\begin{tabular}{|c|c|c|c|c|c|}
\hline \multirow{2}{*}{ Champs } & \multirow{2}{*}{ Critères de sélection } & \multirow{2}{*}{$\begin{array}{c}\text { V_pivot } \\
\text { en français }\end{array}$} & \multirow{2}{*}{$\begin{array}{c}\text { Équivalent } \\
\text { V_pivot en arabe }\end{array}$} & \multicolumn{2}{|c|}{ Nombre d'occurrences } \\
\hline & & & & EmoBase & ArabiCorpus \\
\hline Colère & $\begin{array}{l}\text { Émotion causée, } \\
\text { ponctuelle, réactive, } \\
\text { de polarité négative }\end{array}$ & énerver & [Saydaba] & 255 & 301 \\
\hline Admiration & $\begin{array}{l}\text { Sentiment interper- } \\
\text { sonnel, duratif et de } \\
\text { polarité positive }\end{array}$ & admirer & [?aSzaba] & & 556 \\
\hline
\end{tabular}

L'étude est centrée sur deux champs d'émotion relevés des neuf champs traités dans Emolex (Diwersy et al., 2014), avec un représentant pour chaque champ. Notre objectif est de faire une analyse contrastive qualitative fine plutôt qu'une classification de tous les V_émotion.

Dans la section suivante, nous proposerons une analyse de la façon dont s'articulent les dimensions sémantiques et les relations syntaxiques spécifiques des associations lexicales à $\mathrm{V} \_$pivot d'émotion. Nous testerons, ainsi, notre hypothèse selon laquelle aux différentes dimensions sémantiques correspondent des structures syntaxiques privilégiées et que les associations des $\mathrm{V}$ _émotion ont ainsi des préférences pour des positions et fonctions syntaxiques spécifiques.

${ }^{6}$ Comme l'indice de spécificité log-likelihood qui met en évidence des structures spécifiques pour chacun des V_émotion. 


\section{DIMENSIONS SÉMANTIQUES ET RELATIONS SYNTAXIQUES SPÉCIFIQUES DES VERBES DE COLÈRE ET D’ADMIRATION}

Nous proposerons, dans cette section, une analyse systématique des structures syntaxiques privilégiées dans lesquelles apparaissent les associations et qui correspondent aux dimensions sémantiques les plus saillantes dans les deux corpus : l'intensité (2.1) et l'aspect (2.2).

\subsection{Intensité}

L'intensité constitue la dimension plus marquante en arabe qu'en français. La comparaison interlangue montre une seule structure récurrente en français pour les deux V_émotion étudiés:

(1) Un professeur que j'admirais particulièrement parce qu'il avait su me transmettre son amour de la langue anglaise avait été un officier SS. (Le Monde, 2007)

(2) La lune de miel qu'il entretient avec l'opinion l'énerve prodigieusement. (Libération, 2007)

Comme l'indiquent les exemples [1] et [2], la seule construction syntaxique utilisée pour véhiculer l'intensité forte est «pivot verbal+modifieur adverbial» (V+Adv). En [2], l'attirance exclusive de énerver avec prodigieusement, pourrait susciter une étude précise sur les contextes génériques ou stylistiques d'apparition. En effet, prodigieusement relève d'un lexique soutenu, rare et littéraire. Il s'agit, d'autre part, d'un terme qui entretient une relation antonymique avec le verbe énerver, ce qui en fait un usage proche de l'ironie. On pourrait associer l'emploi de cette combinaison à une forme de routine (Nemo, 2010) journalistique privilégiant un style littéraire. Les collocatifs intensifs ne sont pas les mêmes pour chacun des $V$ émotion, en français. Néanmoins, pour ces patrons, la construction syntaxique «V_émotion + Adv 》 est la seule construction impliquée dans l'expression de l'intensité, ce qui corrobore les conclusions de Novakova, Melnikova et Goossens, (2012) selon lesquelles cette dimension est véhiculée exclusivement par des modifieurs adverbiaux.

Du point de vue contrastif, l'arabe présente une configuration différente des structures syntaxiques. Tout d'abord, c'est la structure $(V+A d j)$ qui est la plus fréquente pour l'expression de l'intensité ([Payḍaba kaقīran] (énerver dense). La structure (V+Nom accusatif) dans laquelle les modifieurs nominaux sont des intensifieurs du sentiment a été aussi relevée ([Pa९zaba ziddan] (admirer sériosité)). Nous avons identifié également des structures composées, à savoir celles composées

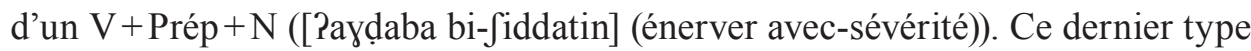


de combinatoire indique une émotion de colère ressentie "d'une manière sévère», ce qui le différencie du fonctionnement lexical des combinaisons verbales d'émotion en français, où l'on n'emploie presque jamais cet $\mathrm{Adv}^{7}$ en combinaison avec un V_émotion. L'adverbe sévèrement a la valeur de de façon sévère, durement, lourdement, rigoureusement. Ces Adv ne trouvent pas de correspondants parfaits en arabe, ce qui implique la tendance à utiliser le $\mathrm{V}$ pivot avec un collocatif libre génitif ([bi-fiddatin] (avec-sévérité)).

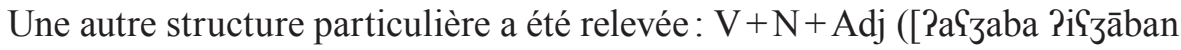
kabīran] (admirer admiration (n. masc.) grande (adj. masc.) :

(3) Orig. translit.: [yaḍaba bin yūrjūn yaḍaban Jadīdan Galā sijāsati Pa-lkitābi Pal-Pabjaḍ] $3^{\mathrm{e}}$ pers. sing $\mathrm{N}$ - marque de l'accusatif Adj - marque de l'accusatif

Trad. litt.: ([il]s'est-énervé ben gourion énervement sévère (Objet absolu accusatif) sur politique de le-livre le-blanc)

Trad. fr.: Ben Gourion s'énerve au plus haut point à cause du livre blanc.

L'exemple [3], qui représente la structure $\mathrm{V} \_$émotion $+\mathrm{N}$ _émotion accusatif déverbal+Adj accusatif, indique tout particulièrement le degré de l'intensité de colère ressentie par l'expérienceur, et ce, en renforçant le sens du V_émotion. Le N_émotion déverbal [yaḍaban] (énervement) se réalise au moyen du déverbal dérivé du V_émotion (Payḍaba] (énerver). Le N déverbal (appelé [masdar]), en l'occurrence le N_émotion, ici, est conçu chez Mel'čuk (1994) comme étant une catégorie flexionnelle syntaxique du verbe dont il est dérivé, voire d'une forme fléchie du verbe. Il s'agit d'une emphase, voire de l'exagération dans l'expression de l'intensité de l'émotion, qui correspond à la nature expressive de la langue arabe ${ }^{8}$. Cela est valable aussi pour le V_[?ązaba] (admirer):

(4) Orig. translit: [?-aYtaqidu Panna kaOīran mina Pal-muslamīna Pal-muhāzirna Pilā Pal-

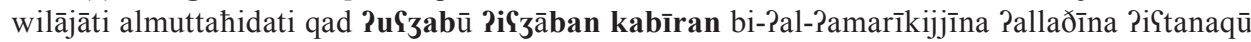
Pal-Pislām]

Accompli - $3^{\text {ème }}$ pers. plur . $\mathrm{N}$ - marque de l'accusatif Adj-marque de l'accusatif

Trad. litt.: (Je crois que beaucoup de les-musulmans les-immigrés à les-états les-unies admirent admiration grande avec les-américains qui convertissent le-islam)

${ }^{7}$ On trouve l'adverbe sévèrement en français, notamment avec des verbes comme: critiquer, juger, châtier, réprimer, sanctionner, condamner, réprimander, endommager, traiter, pénaliser, épingler, traiter, gronder, avertir, regarder, dévisager, réprouver, dire, défendre.

${ }_{8}$ Toutefois, en français, si le nom porte un suffixe, il est morphologiquement déverbal (ex.: trier > triage; laver > lavage). Mais, souvent, il n'y a qu'un radical nu (ex.: le cri, le vol, le port, le manque, etc.). Parfois, on a une paire avec/sans suffixe et deux sens différents (ex. : le tri/triage; le manque/manquement; le port/portage, etc.). En l'absence de suffixe, on hésite alors entre une dérivation du $\mathrm{V}$ ou du $\mathrm{N}$, et on peut considérer que le radical est syntaxiquement neutre et prend sa valeur syntaxique par la combinaison avec un sujet ou un déterminant. 
Trad. fr.: Je pense que de nombreux immigrants musulmans aux États-Unis admirent au plus haut point les Américains qui se convertissent à l'islam.

Cette structure de type « $\mathrm{V} \_$admirer $+\mathrm{N}$ déverbal» constitue une redondance des deux éléments de la même famille sémantique, dans laquelle on peut trouver des associations avec des collocatifs qui sont «non colorés», d'un point de vue de l'intensité de l'émotion (Gross, 1995) (*admirer admiration grande/ *énerver énervement grand). Toutefois, du point de vue contrastif, l'équivalent fonctionnel pourrait être une construction à V_support en français (ex. porter une admiration extrême à qqn).

S'agit-il donc des structures binaires au sens de Hausmann (1989) ou ternaires? Plusieurs raisons peuvent expliquer cette interrogation. D'une part, le système dispose d'une expression particulière, ce qui doit être une spécificité de l'arabe (Al-Sanûsî, 2012). D'autre part, ces structures ternaires peuvent constituer un «enchâssement» de deux collocations, ce qui peut remettre en cause le statut strictement binaire des collocations (Hausmann, 1989, 2007).

Après avoir examiné les spécificités des associations lexicales véhiculant l'intensité, nous proposerons d'étudier, dans la section suivante, les particularités des associations qui véhiculent l'aspect.

\subsection{L'aspect}

L'expression de l'aspect lexical se produit au moyen des constructions syntaxiques spécifiques, qui expriment les valeurs aspectuelles ponctuelle, durative ou phasique. Cette dimension est plus importante en français qu'en arabe, ce qui pourrait s'expliquer par la spécificité de la langue arabe qui, contrairement au français, dispose de marque morphologique spécifique pour l'aspect grammatical. Le français, toutefois, n'a pas de morphèmes aspectuels et a besoin d'accompagnateurs aspectuels autour des verbes pour spécifier la façon dont se déroule le procès.

Pour le V_pivot énerver et son correspondant [Yayḍaba], les associations expriment des valeurs ponctuelles itératives et ce, dans les deux langues:

(5) Le maniérisme du chorégraphe, dont le style néoclassique s'énerve parfois au point de paraître étriqué, réserve des scènes étonnantes. (Le Monde, 2008)

(6) Orig. translit.: ['urīdu 'an 'uhașșiṣa hād̄ā 'almaqāl lilhadīti Pan ru'asā' Pattahrīr fanahnu nayḍabu minhum ahyānan lākin fồ 'ițāri «'alhususūṣiyyati 'al'urduniyyati»]

$\mathrm{V}$ pronominal $1^{\text {re }}$ pers. pluriel présent $\mathrm{N}$ accusatif

Trad. litt.: (je veux consacrer ce le-article pour-la-parole sur chefs le-édition, car-nous nous-énervons de-eux instants, mais dans cadre "la-intimité la-jordanienne»)

Trad. fr.: Je veux consacrer cet article à parler des éditeurs; nous nous énervons parfois contre eux, mais c'est dans le cadre de la «confidentialité jordanienne». 
Ces associations expriment avantage la valeur ponctuelle itérative étant donné qu'elles renvoient à des émotions causées, réactives et ponctuelles (Mathieu, 2000; Ruwet, 1994).

Quant aux verbes admirer et son correspondant [Pa९zaba], ils s'associent préférentiellement à des accompagnateurs duratifs.

(7) Le personnage du clown m'est venu spontanément mais, rétrospectivement, je peux dire que j'ai toujours admiré les clowns, parce qu'ils sont proches de cette très particulière ouverture d'esprit et de cœur qu'ont les enfants jusque vers 7 ans. (Libération, 2007)

(8) Orig. translit. : [?anā - mutaћajjizatun ziddan, li-Pa-ssinimā wa li-ðālika Puৎzabu dāPiman bi-kolli PaSmāli zumalā-Pī]

$\mathrm{V}$ tansi $1^{\text {re }}$ pers. sing. présent $\mathrm{N}$ accusatif

Trad. litt.: (moi- impliqué sériosité à le-cinéma et pour-cela je-admire qui-dure avectout travaux collègues-moi)

Trad. fr. : J'ai une attitude impartiale à l'égard du cinéma et c'est pour cela que j'admire toujours tous les travaux de mes collègues.

Il s'agit, ici, d'une insistance sur la durée du sentiment d'admiration au moyen de «toujours». L'admiration (qui est un sentiment duratif) dure dans le temps. Le procès «est appréhendé dans un développement interne : il a un début et il ne prend absolument pas en compte un quelconque dernier instant» (Desclés, 1994: 80).

Néanmoins, l'étude de ce modifieur en contexte montre qu'il est polysémique. Il peut pencher plutôt du côté de l'aspect ponctuel ${ }^{9}$ tout en acceptant en même temps des emplois duratifs, qui indiquent que l'expérienceur poursuit dans le sentiment ressenti :

(9) Ça m'énerve toujours qu'on définisse une ligne politique par rapport à des choix personnels, comme ceux qu'ont faits Jean-Luc Bennahmias et Yann Wehrling. (Le Figaro, 2008)

En [9], le locuteur ne ressent pas la colère durant la totalité du temps, mais seulement à chaque fois qu'on relie la définition politique au choix personnel. Il correspond, ici, à la notion d'un ressenti de colère dont la reprise est discontinue ${ }^{10}$.

Ces tendances illustrent bien la relation entre les valeurs aspectuelles et les structures syntaxiques spécifiques.

${ }^{9}$ Cf. Le Grand Robert qui donne l'exemple suivant, marquant la coïncidence avec une circonstance: «Il grognait toujours, quand...» pour désigner chaque fois que.

${ }^{10}$ Dans l'exemple suivant, issu du Grand Robert: «Il arrive toujours à cinq heures », nous n'avons aucune idée de durée, mais de reprise ponctuelle du procès. Et aussi dans un autre exemple du Grand Robert: «Il est en retard, lui toujours ponctuel», l'adverbe toujours signifie qu'à chaque fois il est ponctuel. 


\section{CONCLUSION}

Nous avons mis en évidence, dans ce travail, les dimensions sémantiques statistiquement importantes (l'intensité et l'aspect), en français et en arabe. Les résultats obtenus valident notre hypothèse de départ, qui supposait que les liens syntaxiques spécifiques (les constructions grammaticales récurrentes) de ces associations lexicales sont étroitement liés aux dimensions sémantiques qu'elles véhiculent (Hoey, 2005 ; Novakova et al., 2012). L'analyse du profil combinatoire de chaque V_émotion permet l'étude de «l'ensemble de ses accompagnateurs stéréotypés, porteurs d'associations typiques» (Blumenthal, 2007). Il est nécessaire de poursuivre ce travail par l'étude d'un plus grand ensemble de V_émotion, et ce, dans une perspective contrastive.

\section{RÉFÉRENCES BIBLIOGRAPHIQUES}

Abu-Ssaydeh, A. (2007). Collocations and the Arabic-English dictionary. Ideas for better dictionaries. International Journal of Arabic-English Studies (IJAES), 8, 69-90.

Arbaoui, N. (2010). Les dix formes de l'arabe classique à l'interface syntaxe/phonologie-Pour une déconstruction du gabarit, thèse de doctorat, Université Paris 7 - Denis-Diderot.

Blumenthal, P. (2007). Profil combinatoire des mots: analyse contrastive. In: C. Bolly, J.R. Klein, B. Lamiroy (éds.). La phraséologie dans tous ses états. Actes du colloque Phraséologie 2005 (Louvain-la-Neuve, 13-15 octobre 2005) (pp. 131-148). Louvain-la-Neuve: Université catholique de Louvain.

Bouchard, D. (1995). Les verbes psychologiques. Langue française, 105, 6-16.

Creissels, D. (1995). Éléments de syntaxe générale. Paris: Presses universitaires de France.

Desclès, J.-P. (1994). Quelques concepts relatifs au temps et à l'aspect. Studia Kognitywne. Semantyka kategorii aspektu i czasu, 1, 57-88.

Diwersy, D., Goossens, V., Grutschus, A., Kern, B., Kraif, O., Melnikova, E., Novakova, I. (2014). Traitement des lexies d'émotion dans les corpus et les applications d'EmoBase. Corpus, 13, 269-293.

Donat Vernier, S.J. (1892). Grammaire arabe composée d'après les sources primitives. T. 2. Beyrouth: Imp. catholique.

Dubois, J., Dubois-Charlier, F. (1997). Les Verbes français. Paris : Larousse.

El Kassas, D. (2007). Vers une typologie des équivalents structuraux arabes de l'infinitiffrançais. Conférence internationale de la Faculté de Langue et traduction (Al Alsun), Université de Minia, 23-25 avril 2007, Minia.

Gross, M. (1995). Une grammaire locale de l'expression des sentiments. Langue française, 105, $70-87$.

Harris, Z.S. (1988). Language and Information. New York: Columbia University Press.

Hausmann, F. J. (1989). Le dictionnaire de collocations. In: F. J. Hausmann, O. Reichmann, H.E. Wiegand, L. Zgusta (eds.), Worterbucher: ein nternationales Handbuch zur Lexicographie. Dictionnaries, Dictionnaires (pp. 1010-1019). Berlin/New-York: De Gruyter. 
Hausmann, F.J. (2007). Lexicologie française et phraséologie. Collocation, phraséologie, lexicographie, Aachen: Shaker Verlag, 121-53.

Hoey, M. (2005). Lexical priming : a new theory of words and language. London/New York: Routledge.

Ibrahim, A.H. (2001). Une classification des verbes en six classes asymétriques hiérarchisées. Syntaxe et sémantique, 1(2), 81-97. Https://doi.org/10.3917/ss.002.0081 [Article disponible en ligne à l'adresse: http://www.cairn.info/revue-syntaxe-et-semantique-2001-1-page-81.htm (dernière consultation le 11 octobre 2018)].

Mathieu, Y.Y. (2000). Les verbes de sentiment: de l'analyse au traitement automatique. Paris: Éditions du CNRS.

Mel’čuk, I. (1994). Cours de morphologie générale. Vol. 2. Montréal: Presses de l'université de Montréal, CNRS Éditions.

Nemo, Fr. (2010). Routines interprétatives, constructions grammaticales et constructions discursives. Estudos Linguísticos/Linguistic Studies, 5, 35-53.

Novakova, I., Goossens, V., Melnikova, E. (2012). Associations sémantiques et syntaxiques spécifiques. Sur l'exemple du lexique émotionnel des champs de surprise et de déception. In: F. Neveu, V. Muni Toke, P. Blumenthal, T. Klingler, P. Ligas, S. Prévost, S. Teston-Bonnard (éd.), Actes du $3^{e}$ Congrès mondial de linguistique française. Lyon, 4-7 juillet 2012 (pp. 1017-1029). Les Ulis, France: EDP Sciences.

Novakova, I., Tutin, A. (éd.) (2009). Le lexique des émotions. Grenoble: ELLUG.

Ruwet, N. (1972). Théorie syntaxique et syntaxe du français. Paris: Seuil.

Ruwet, N. (1994). Être ou ne pas être un verbe de sentiment. Langue française, 103, 45-55.

Sinclair, J.M. (1991). Corpus, concordance, collocation. Oxford: Oxford University Press (Describing English Language).

The Leipzig Glossing Rules : Conventions for interlinear morpheme-by-morpheme glosses, https:// www.eva.mpg.de/lingua/pdf/Glossing-Rules.pdf [consulté le 27 septembre 2017].

\section{RÉSUMÉ}

L'étude concerne les correspondances entre le français et l'arabe dans l'expression de l'émotion. Certains chercheurs proposent de classifier les verbes d'émotion en s'appuyant sur des critères syntaxiques (Harris, 1988). D'autres partent, à l'inverse, d'ensembles sémantiquement similaires de verbes pour aborder leurs structures syntaxiques (Mathieu, 2000). Nous proposons, ici, une troisième voix dont l'objectif consiste à mieux appréhender les spécificités de la combinatoire des V_d'émotion à travers la structuration de ce champ sémantique dans les deux langues par rapport à ce que propose la littérature linguistique en syntaxe et en sémantique contrastive. Nous examinerons, ainsi, les aspects sémantico-syntaxiques de ces associations pour savoir, si des verbes appartenant aux mêmes types d'affect dans les deux langues attirent les mêmes accompagnateurs ou non. Nous choisissons de recourir à la combinatoire syntaxique et lexicale (Novakova et Tutin, 2009), qui permet d'identifier les associations lexicales relevées en français en arabe. Le travail s'appuie sur la théorie du Lexical priming de Michael Hoey (2005) qui permet d'examiner les relations syntaxiques privilégiées des combinaisons verbales d'émotion et de vérifier si elles sont liées aux dimensions sémantiques qu'elles peuvent éventuellement véhiculer. Après une présentation du cadre théorique, de l'approche contrastive et de la méthodologie, ainsi que des corpus (Emolex et Arabicorpus), nous proposerons une analyse sémantico-syntaxique de ces associations et ce, dans les deux langues comparées.

Mots-clés : combinatoire verbale, analyse sémantico-syntaxique, approche contrastive 


\begin{abstract}
The study concerns the correspondences between French and Arabic in the expression of emotion. For the verbs of emotion, some authors offer a classification based on syntactic criteria (Harris, 1988). Others propose a distribution of verbs based on homogeneous semantic features (Mathieu, 2000). Proposing a third way, our objective is to better understand the specificities of the verbal combining of emotion in French and Arabic through the structuring of this semantic field in both languages in relation to what is proposed in linguistic, literature, syntax and contrasting semantics. We examine syntactic-semantic correspondences in the verbal combinations between the two languages seeking to know if verbs belonging to the same types of emotion in the two languages attract the same companions. We choose to base our study on the analysis of syntactic and lexical combination (Novakova, Tutin, 2009), which allow us to identify the combination of V_emotion studied in the two compared languages. We use Lexical priming theory (Hoey, 2005) to examine the preferred syntactic relationships of the verbal combination of emotion and to verify whether they are linked to the semantic dimensions that they can possibly convey. We show that semantics is systematically connected to syntax. After presenting the theoretical framework, the contrastive approach and the methodology of the work, and the corpus on which this research was based (Emolex and Arabicorpus), we propose a semantic-syntactic analysis of the combinations of emotion verbs belonging to the lexical fields of anger and admiration, in the two compared languages.
\end{abstract}

Keywords: verbal combinations, semantic-syntactic analysis, contrastive analysis

\begin{abstract}
ABSTRAKT
Celem artykułu jest pokazanie podobieństw w wyrażaniu emocji w języku francuskim i arabskim na przykładzie czasowników odnoszących się do stanów emocjonalnych. Niektórzy lingwiści proponują klasyfikację wymienionych leksemów opartą na kryteriach syntaktycznych (Harris, 1988), inni - ich podział na podstawie homogenicznych cech semantycznych (Mathieu, 2000). Biorąc pod uwagę dotychczasową literaturę przedmiotu w obrębie składni i semantyki kontrastywnej, w badaniach skoncentrowano się na ukazaniu specyfiki łączliwości interesujących nas czasowników przez analizę sposobu ustrukturyzowania pola semantycznego nazw emocji w obu porównywanych językach. Opisano zatem syntaktyczną i leksykalno-semantyczną łączliwość czasowników (Novakova, Tutin, 2009), aby dowiedzieć się, czy leksemy czasownikowe, które nazywają ten sam rodzaj emocji w języku francuskim i arabskim, współwystępują z ekwiwalentnymi wyrazami. Pozwoliło to zidentyfikować połączenia, w skład których wchodzą czasowniki emocji w porównywanych językach. W artykule odwołano się do teorii primingu leksykalnego (Hoey, 2005), aby zbadać preferowane relacje syntaktyczne w połączeniach z czasownikami emocji i zweryfikować, czy są one powiązane z takimi parametrami semantycznymi, jak intensywność czy aspekt. Pokazano, że semantyka jest systemowo powiązana ze składnią. Po przedstawieniu ram teoretycznych analizy kontrastywnej i metodologii pracy oraz scharakteryzowaniu korpusu, na którym oparto badania (Emolex i Arabicorpus), zaproponowano semantyczno-syntaktyczną analizę związków łączliwych, należących do pól semantycznych colère (gniew, złość) oraz admiration (podziw) w języku francuskim i arabskim.
\end{abstract}

Słowa kluczowe: łączliwość czasowników emocji, analiza semantyczno-syntaktyczna, analiza kontrastywna 